\title{
(6) OPEN ACCESS \\ Safety, tolerability and potential efficacy of injection of autologous adipose-derived stromal vascular fraction in the fingers of patients with systemic sclerosis: an open-label phase I trial
}

\author{
Brigitte Granel, ${ }^{1,2}$ Aurélie Daumas, ${ }^{1}$ Elisabeth Jouve, ${ }^{3}$ Jean-Robert Harlé, ${ }^{1}$ \\ Pierre-Sébastien Nguyen, ${ }^{4}$ Christian Chabannon, ${ }^{5}$ Nathalie Colavolpe, ${ }^{6}$ \\ Jean-Charles Reynier, ${ }^{7}$ Romain Truillet, ${ }^{3}$ Stéphanie Mallet, ${ }^{8}$ Antoine Baiada, ${ }^{4}$ \\ Dominique Casanova ${ }_{1}^{4}$ Laurent Giraudo, ${ }^{9}$ Laurent Arnaud,${ }^{10}$ Julie Veran ${ }^{9}$ \\ Florence Sabatier, ${ }^{2,9,10}$ Guy Magalon ${ }^{4,9}$
}

\section{Handling editor Tore K Kvien \\ - Additional material is published online only. To view please visit the journal online (http://dx.doi.org/10.1136/ annrheumdis-2014-205681). \\ For numbered affiliations see end of article. \\ Correspondence to Professor Brigitte Granel, North Hospital, Assistance Publique Hôpitaux de Marseilles (AP-HM), Marseilles 139015, France; bgranel@ap-hm.fr}

Received 4 April 2014 Revised 15 July 2014 Accepted 20 July 2014 Published Online First 11 August 2014

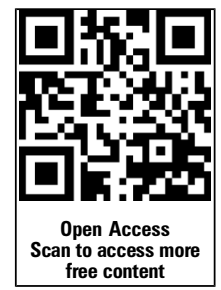

To cite: Granel $B$, Daumas $A$, Jouve $E$, et al. Ann Rheum Dis

2015:74:2175-2182.

\section{ABSTRACT}

Background In patients with systemic sclerosis (scleroderma, SSc), impaired hand function greatly contributes to disability and reduced quality of life, and is insufficiently relieved by currently available therapies. Adipose tissue-derived stromal vascular fraction (SVF) is increasingly recognised as an easily accessible source of regenerative cells with therapeutic potential in ischaemic or autoimmune diseases. We aimed to measure for the first time the safety, tolerability and potential efficacy of autologous SVF cells local injections in patients with SSC with hand disability.

Methods We did an open-label, single arm, at one study site with 6-month follow-up among 12 female SSc patients with Cochin Hand Function Scale score $>20 / 90$. Autologous SVF was obtained from lipoaspirates, using an automated processing system, and subsequently injected into the subcutaneous tissue of each finger in contact with neurovascular pedicles. Primary outcome was the number and the severity of adverse events related to SVF-based therapy. Secondary endpoints were changes in hand disability and fibrosis, vascular manifestations, pain and quality of life from baseline to 2 and 6 months after cell therapy.

Findings All enrolled patients had surgery, and there were no dropouts or patients lost to follow-up. No severe adverse events occurred during the procedure and follow-up. Four minor adverse events were reported and resolved spontaneously. A significant improvement in hand disability and pain, Raynaud's phenomenon, finger oedema and quality of life was observed.

Interpretation This study outlines the safety of the autologous SVF cells injection in the hands of patients with SSc. Preliminary assessments at 6 months suggest potential efficacy needing confirmation in a randomised placebo-controlled trial on a larger population.

Funding GFRS (Groupe Francophone de Recherche sur la Sclérodermie).

Clinical Trials number NCT01813279.

Systemic sclerosis (scleroderma, SSc) is a rare systemic autoimmune disease characterised by microvascular lesions and progressive fibrosis of skin and internal organs. Hands involvement is the most classical and visible manifestation of the disease and is a large burden on global disability and social relationship. Hand disability has multifactorial origins with microvascular lesions, paroxysmal vasospasm or permanent ischaemia and subsequently digital ulcers (DU), skin sclerosis, tendons retractions, bone and articular involvement, and subcutaneous calcinosis. ${ }^{1-3}$ In patients with SSc, radiological abnormalities included erosion (21\%), joint space narrowing (28\%), arthritis (18\%), acro-osteolysis (22\%), flexion contracture (27\%) and calcinosis (23\%). ${ }^{4}$ All these lesions cause pain, functional impairment, aesthetic and psychological distress. ${ }^{1}$ To date, therapeutic interventions for SSc hands have mainly focused on vascular manifestations, including Raynaud's phenomenon and DUs. ${ }^{5-9}$ These treatments are often life-long therapies with considerable costs and potentially significant side effects. No antifibrotic drug is effective in SSc. Conventional immunosuppressive drugs effectively treat joint synovitis and sometimes even improve skin fibrosis but do not improve contractures of small joints. ${ }^{10}$

Most research priorities are aimed to prevent or treat life-threatening complications that arise from affection of internal organs; however, if asked, patients indicate that hand disability interfering with daily life and work matters most. ${ }^{11}$ Thus, healthcare professionals should consider improving disability, patients' satisfaction and social comfort as clinically relevant objectives of therapy.

The delivery of autologous stem/progenitor cells into ischaemic tissue of patients is emerging as a novel therapeutic option for vascular diseases. To date, bone marrow-derived cells have mainly been used in such context ${ }^{12-14}$ but are limited by the invasive nature of bone marrow harvest, the delay and quality management issues associated with cells culture. ${ }^{15}$ In 2002, Zuk and colleagues ${ }^{16}$ characterised a multipotent population of cells within the stromal vascular fraction (SVF) of adipose tissue, referred to adipose-derived stromal/stem cells (ADSC). In addition to multipotency, this population exerts paracrine proangiogenic effects through the increased expression of vascular endothelial 
growth factor, basic fibroblast growth factor and interleukin- $6 .{ }^{17}$ Moreover, these cells have anti-inflammatory effects that appear to be mediated through secretion of anti-inflammatory soluble factors such as interleukin-10, interferon- $\gamma$, transforming growth factor- $\beta$ or prostaglandin E2, with dendritic cells immunomodulation, stimulation of regulatory $\mathrm{T}$ cells and inhibition of inflammatory cytokines synthesis. ${ }^{18}$

Results from the recent ACellDREAM ${ }^{19}$ trial demonstrated the safety of autologous cultured ADSC in patients with critical limb ischaemia, with favourable secondary endpoints on efficacy. Recently, six patients with localised scleroderma (linear and plaque scleroderma, generalised morphea) have been locally treated with in vitro expanded autologous ADSC with a favourable safety profile and a significant improvement in the skin, at 1-year follow-up. ${ }^{20}$

Thus, adipose tissue-derived SVF may have potential efficacy against ischaemia and skin fibrosis, two major manifestations of SSc. We tested in this early phase clinical trial the safety and tolerability of local injections of autologous SVF cells in fingers and assessed preliminary efficacy outcomes in patients with SSc with diminished hand function.

\section{METHODS}

\section{Study design and eligibility criteria}

Twelve patients with SSc were enrolled from December 2012 through May 2013 in an open-label, single-arm and single study site. All subjects fulfilled the 1980 American College of Rheumatology criteria ${ }^{21}$ or the 1988 LeRoy et al criteria. ${ }^{22}$ All enrolled patients had a score of $\geq 9$ according to the 2013 Classification criteria for SSc. ${ }^{23}$ Subjects had previously received optimal treatment for digital vasculopathy, according to EULAR. ${ }^{5}$

Patients were eligible if they were aged $>18$ and had a Cochin Hand Function Scale (CHFS) ${ }^{24}>20 / 90$. Exclusion criteria were new vasodilators or immunosuppressive therapy for SSc in the 3 months prior to enrolment or during the 6-month follow-up, surgical contraindication, clinical or radiological signs of digital infection, positive status for HIV, hepatitis B or C, HTLV1-2 or syphilis, pregnancy and body mass index $<17 \mathrm{~kg} / \mathrm{m}^{2}$.

Patients were asked not to change their regular medications and physiotherapy of the hands during the trial. The study was approved by the local Ethics Committee and the French Drug Agency. The study was done in accordance with the Declaration of Helsinki and current Good Clinical Practices. An independent data monitoring committee ensured the integrity of the trial and safety of participants. All patients gave written informed consent before participation. This study is registered with ClinicalTrials.gov number NCT01813279.

\section{Procedures}

Tissue collection and SVF preparation

Adipose tissue collection and SVF injection were conducted under conscious sedation; harvesting areas were anaesthetised. Harvesting was performed with a $10 \mathrm{~mL}$ syringe in a closed circuit using a $3 \mathrm{~mm}$ Coleman cannula with 2 terminal $4 \mathrm{~mm} \times 2 \mathrm{~mm}$ openings with a two-way non-return AT valve, sterile tubing and a $250 \mathrm{~mL}$ collection bag. Once harvesting was complete, the bag was immediately transported to the registered Cell Therapy Unit. SVF was obtained within $2 \mathrm{~h}$ after lipoaspiration using the automated processing Celution800/CRS system (Cytori Therapeutics, San Diego, California, USA). Collected lipoaspirate was washed and enzymatically digested using GMP-grade reagents. Cells were concentrated, washed, aseptically recovered and resuspended in $5-11 \mathrm{~mL}$ Lactate Ringer's solution. The final cell suspension was transferred into 10 syringes $(1 \mathrm{~mL})$ for injection. The remaining volume was used for sterility testing and biological characterisation. Total viable nucleated cell recovery and viability percentage were determined using the Nucleocounter NC100 (ChemoMetec, Denmark). Cellular components were identified by flow cytometry analysis (Beckman Navios instrument) with a panel of cell surface makers in agreement with International Federation for Adipose Therapeutics and Science (IFATS) and the International Society for Cellular Therapy (ISCT) recommendations. ${ }^{25}$ The following markers (CD45, CD34, CD90, CD146, CD14) were used in combination with DRAQ5 and DAPI to exclude debris, red blood cells and dead cells. The frequency of adipose-derived mesenchymal-like stem cells was estimated using the Colony-Forming-Unit-Fibroblastic clonogenic assay. ${ }^{25}$

\section{SVF delivery}

Entry points were preformed using a 25 -gauge needle $(0.5$ $\mathrm{mm} \times 16 \mathrm{~mm})$. SVF cells were then injected using a 25 -gauge $(0.5 \mathrm{~mm} \times 40 \mathrm{~mm})$ reinforced cannula placed into the subcutaneous tissue in contact with the neurovascular pedicles: $0.5 \mathrm{~mL}$ SVF was injected into each lateral side of each digit, using a retro-tracing technique, from distal to proximal. Entry points were positioned at the metacarpophalangeal joint for the thumb, and the proximal interphalangeal joint where the palmar and dorsal skin joins for long fingers. Both hands were treated over a period of approximately $20 \mathrm{~min}$.

Assessment of safety, tolerability and efficacy

See online supplementary table $\mathrm{S} 1 .^{26-30}$

\section{Statistical analysis}

See online supplementary text.

\section{RESULTS}

\section{Characteristics of the patients and the SVF}

All enrolled patients had surgery, and there were no dropouts or patients lost to follow-up. The population mainly included patients with limited cutaneous form of the disease and without severe organ damage (table 1). No patient was treated with $>10 \mathrm{mg}$ steroids daily, cyclophosphamide or mycophenolate mofetil. Hand radiology revealed abnormalities in 10/12 patients (see online supplementary table S2). No patient had radial or ulnar occlusion on arterial Doppler ultrasound.

Patients received a mean of $3.76 \pm 1.85 \times 10^{6}$ viable cells into each finger. The infused cells contained a mean of $3.7 \pm 1.9 \%$ CFU-F. Flow cytometry characterisation of cell subsets in SVF is shown in table 1.

\section{Safety and tolerance profile of autologous adipose-derived SVF injection}

All patients were discharged from hospital $24 \mathrm{~h}$ after surgery. No serious AEs occurred during follow-up. Four minor AEs reported by four patients were potentially related to the procedure: two abdominal bruises induced by the lipoaspiration of respectively 7 and 15 days duration, one transient paresthesia on the lateral side of the left fifth finger persisting for 11 days postinjection and one pain located on the lateral side of the left thumb persisting for 13 days postinjection. These events spontaneously resolved. Abdominal lipoaspiration sample points healed in less than 7 days postsurgery and the points of entry for SVF injection healed as soon as $\mathrm{D}+1$. Abdominal pain remained moderate and transient: visual analogue scale (VAS) of $32 \pm 13$ on $\mathrm{D}+1,47 \pm 18$ 
Table 1 Characteristics of the 12 patients with systemic sclerosis (SSc) and adipose tissue harvest

\begin{tabular}{|c|c|}
\hline Demographic & \\
\hline Number of patients, sex & 12, all female \\
\hline Age (years) & $54.5 \pm 10.3(34.0-68.0)$ \\
\hline Body mass index $\left(\mathrm{kg} / \mathrm{m}^{2}\right)$ & $22.0 \pm 2.1(18.6-26.2)$ \\
\hline \multicolumn{2}{|l|}{ Dominant hand } \\
\hline Right-handed/left-handed & $10(83.3 \%) / 2(16.7 \%)$ \\
\hline \multicolumn{2}{|l|}{ Main causes of hand disability } \\
\hline 1. Vascular aspects (Raynaud's phenomenon) & 12 patients $(100 \%)$ \\
\hline 2. Skin fibrosis (sclerodactyly) & 11 patients $(92 \%)$ \\
\hline 3. Musculoskeletal aspects (joint synovitis) & 5 patients $(42 \%)$ \\
\hline \multicolumn{2}{|l|}{ Cardiovascular risk factors } \\
\hline Tobacco & $0(0.0 \%)$ \\
\hline Diabetes & $0(0.0 \%)$ \\
\hline Arterial hypertension & $0(0.0 \%)$ \\
\hline \multicolumn{2}{|l|}{ Disease history } \\
\hline Disease duration from diagnosis (years) & $9.9 \pm 7.0(2.0-24.0)$ \\
\hline Early SSC disease $<4$ years & $3(25 \%)$ \\
\hline History of Raynaud's phenomenon (years) & $14.3 \pm 7.7(5.0-34.0)$ \\
\hline Systemic sclerosis cutaneous subclassification: limited/diffuse & $7(58.3 \%) / 5(41.7 \%)$ \\
\hline Pulmonary arterial hypertension or previous renal crisis & $0(0.0 \%)$ \\
\hline \multicolumn{2}{|l|}{ Rodnan } \\
\hline Global modified Rodnan skin score & $13.9 \pm 9.8(3-32)$ \\
\hline \multicolumn{2}{|l|}{ Gastrointestinal } \\
\hline Gastrointestinal reflux & $9(75 \%)$ \\
\hline Hypotonia of the inferior oesophagus sphincter (manometry) & 9/9 tested \\
\hline \multicolumn{2}{|l|}{ Lung } \\
\hline Total lung capacity $<80 \%$ & $0(0 \%)$ \\
\hline Lung capacity for carbon monoxide/alveolar ventilation $<75 \%$ & $5(41.7 \%)$ \\
\hline Fibrosis on high-resolution chest tomography (bronchiectasia and/or honeycombing) & $2(16.7 \%)$ \\
\hline \multicolumn{2}{|l|}{ Serology } \\
\hline Anti-nuclear antibodies positive (indirect immunofluorescence on HEp2) & $12(100 \%)$ \\
\hline Anti-topoisomerase 1 (Scl70) antibodies positive (ELIA) & $6(50.0 \%)$ \\
\hline Anti-centromere antibodies positive (ELIA) & $4(33.3 \%)$ \\
\hline Anti-SSA antibodies positive (ELIA) & $2(16.7 \%)$ \\
\hline \multicolumn{2}{|l|}{ Ongoing and previous systemic sclerosis medications } \\
\hline Calcium-channel blockers & 6 ongoing (50.0\%), 11 previously (91 7\%) \\
\hline Bosentan & 2 ongoing $(16.6 \%), 4$ previously (33 3\%) \\
\hline Prednisone $<10 \mathrm{mg} /$ day & 3 ongoing $(25.0 \%)$ \\
\hline Methotrexate & 1 ongoing $(8.3 \%)$ \\
\hline lloprost infusion & 0 ongoing, 8 previously $(66.7 \%)$ \\
\hline \multicolumn{2}{|l|}{ Adipose tissue harvest and cell characterisation } \\
\hline Amount of adipose tissue harvest $(\mathrm{mL})$ & $181.3 \pm 50.8(120-270)$ \\
\hline Number of total viable nucleated cells $\left(\times 10^{6}\right)$ & $50.5 \pm 23.8(16.7-92.6)$ \\
\hline$\%$ of cell viability & $88.0 \pm 4.8(81.0-94.6)$ \\
\hline Number of viable nucleated cells injected into each finger $\left(\times 10^{6}\right)$ & $3.76 \pm 1.85(1.19-7.07)$ \\
\hline$\%$ of CFU-F & $3.7 \pm 1.9(1.6-8.1)$ \\
\hline$\%$ of blood leucocytes (CD45 CD34- cells) & $49.1 \pm 18.0$ \\
\hline$\%$ of endothelial progenitor cells (CD34brightCD146dimCD45-) & $3.4 \pm 3.2$ \\
\hline$\%$ of circulating endothelial cells (CD34dimCD146brightCD45-) & $6.4 \pm 6.8$ \\
\hline$\%$ of stromal cells (CD45-CD34brightCD146-CD90+) & $36.0 \pm 14.5$ \\
\hline$\%$ of resident macrophages (CD45+CD14+CD34dim) & $5.1 \pm 2.3$ \\
\hline
\end{tabular}

Data are mean $\pm \mathrm{SD}$ (minimum and maximum) or $\mathrm{n}(\%)$ of patients.

on $\mathrm{D}+7$ and $13 \pm 17$ on $\mathrm{D}+21$, and resolved completely at $\mathrm{M} 2$. Hand pain did not worsen. SVF injection led to a rapid decrease of the Raynaud's Condition Score (RCS) from 7.2 \pm 0.9 at baseline to $5.3 \pm 1.7$ at $\mathrm{D}+7$ and to $4.6 \pm 3.0$ at $\mathrm{D}+2.1$. The number of patients with normal finger colour increased from 5 at baseline to 8 at $\mathrm{D}+7$ and $\mathrm{D}+21$, without colour score worsening.
Efficacy profile of autologous adipose-derived SVF injection Effects on quality of life

Patients perceived their general health status to be improved as shown by a $45.2 \%$ and $42.4 \%$ decrease of the Scleroderma Health Assessment Questionnaire (SHAQ) at M2 ( $p=0.001)$ and at M6 $(\mathrm{p}=0.001)$, respectively (figure 1$)$. 
Figure 1 Six-month follow-up for patients with systemic sclerosis receiving stromal vascular fraction injection ( $p<0.05$ at M2 and M6).

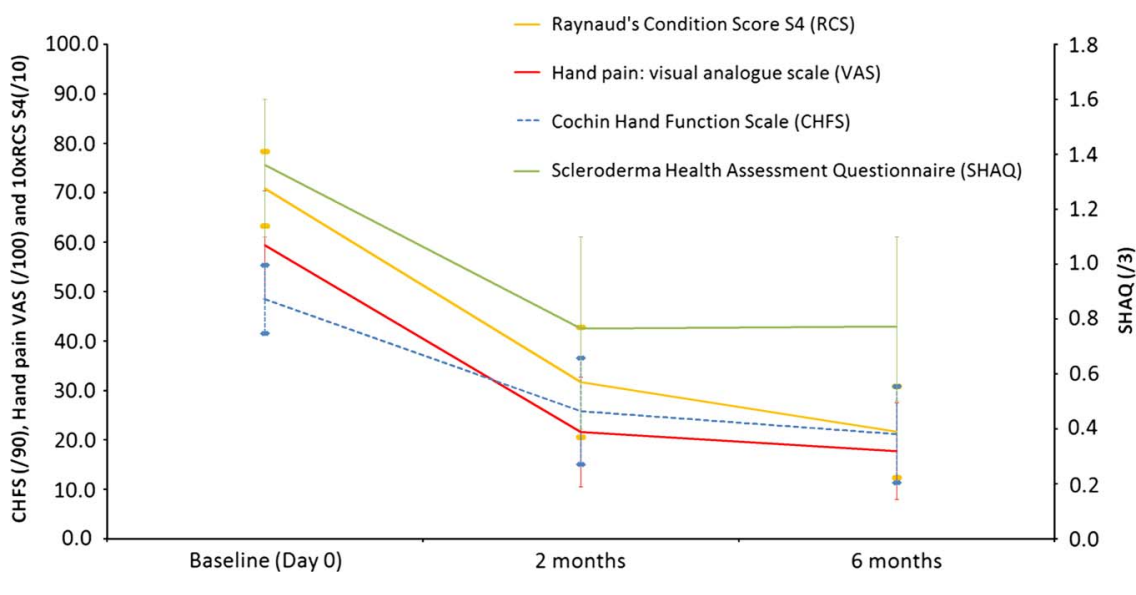

baseline to M6 in both hands ( $\mathrm{p}=0.047$ for both). Finally, a significant decrease in the vascular suppression score was observed from baseline to M6 ( $p=0.01$ for the dominant hand and $\mathrm{p}=0.003$ for the non-dominant hand).

\section{DISCUSSION}

This study demonstrates that injection of autologous SVF from adipose tissue into the fingers of patients with SSc can be performed safely and is well tolerated. The only safety-related events were minor bruising, consistent with what is normally seen following lipoaspiration, and transient finger discomfort. Importantly, a priori efficacy outcomes variables revealed a decrease of $\sim 50 \%$ in CHFS, Raynaud's severity and hand pain at 2 months with persisting benefits at 6 months. To our knowledge, SVF is the first treatment improving such hand manifestations and with favourable impact on quality of life in patients with SSc.

Adipose-derived SVF yields a heterogeneous population of cells including stem and progenitor cells with multipotent differentiation potential. SVF cells transcribe many genes that are implicated inflammation, angiogenesis and tissue repair. ${ }^{31}$ It is suggested that ADSC can have antifibrotic properties by the reduction of local infiltration of inflammatory cells into tissue by the secretion of antifibrotic factors such as interferon- $\gamma$ and matrix metalloproteinases, ${ }^{31}$ and by the decrease of pro-fibrotic factors such as transforming growth factor- $\beta{ }^{32}$ Advantages of SVF include (1) ease of obtaining cells from lipoaspirates, (2) larger pool of ADSC compared with the pool of bone marrowderived mesenchymal stem cells (BM-MSC) and (3) stronger angiogenic and regenerative potential of ADSC compared with BM-MSC. ${ }^{33}$ As a result, the use of SVF cells for tissue regeneration or autoimmune diseases is increasing as shown by numerous ongoing clinical trials (see Clinicaltrial.gov).

The present study mainly enrolled patients with SSc free of severe visceral manifestations but with long disease duration. Some of them had hand flexion contracture, joint space narrowing, severe vascular disease, calcinosis and acro osteolysis. These characteristics may explain the higher frequency of joint involvement and radiographic abnormalities compared with that previously reported by Avouac et al. ${ }^{4}$ Major hand disability in our cohort was also consistent with a CHFS score much higher than that reported by Rannou et al. ${ }^{24}$ Despite such difference, we similarly noticed a strong correlation between CHFS and SHAQ, with the CHFS scores contributing to $71.3 \%$ of the SHAQ variance at M6. This result highlights the crucial contribution of hand disability in global patient disability. Importantly, treatments and physiotherapy schedule were not changed for 
Table 2 Effect of autologous adipose-derived stromal vascular fraction injection on hand disability

\begin{tabular}{|c|c|c|c|c|c|c|c|}
\hline & Baseline & 2 months & Change (2 months-baseline) & p Value* & 6 months & Change (6 months-baseline) & $\mathrm{p}$ Valuet \\
\hline \multicolumn{8}{|l|}{ CHFS total } \\
\hline Mean \pm SD & $48.5 \pm 10.8$ & $25.8 \pm 17.0$ & $-22.7 \pm 16.4$ & $<0.001$ & $21.2 \pm 15.4$ & $-27.3 \pm 17.2$ & $<0.001$ \\
\hline Median (range) & $48.5(30-69)$ & $25.5(2-49)$ & $-22.5(-51-10)$ & & $20.0(0-48)$ & $-30.0(-58-10)$ & \\
\hline \multicolumn{8}{|l|}{ Jamar score (kg) } \\
\hline \multicolumn{8}{|l|}{ Dominant hand } \\
\hline Mean \pm SD & $16.0 \pm 5.8$ & $17.9 \pm 7.3$ & $3.0 \pm 5.0$ & 0.072 & $19.4 \pm 7.4$ & $4.8 \pm 6.4$ & 0.033 \\
\hline Median (range) & $15.0(9.0-26.5)$ & $18.5(5.5-28.0)$ & $2.0(-3.0-15.0)$ & & $20.0(5.0-30.0)$ & $3.0(-6.0-17.0)$ & \\
\hline \multicolumn{8}{|c|}{ Non-dominant hand } \\
\hline Mean \pm SD & $14.9 \pm 6.1$ & $17.5 \pm 7.8$ & $3.7 \pm 3.6$ & 0.006 & $17.6 \pm 8.0$ & $4.0 \pm 3.5$ & 0.002 \\
\hline Median (range) & $14.0(6.0-26.0)$ & $18.5(4.0-28.0)$ & $3.0(-2.0-9.0)$ & & $20.0(3.5-29.0)$ & $3.0(0.0-13.0)$ & \\
\hline \multicolumn{8}{|l|}{ Pinch score $(\mathrm{kg})$} \\
\hline \multicolumn{8}{|l|}{ Dominant hand } \\
\hline Mean \pm SD & $1.3 \pm 1.1$ & $2.0 \pm 0.8$ & $0.6 \pm 1.3$ & 0.199 & $2.3 \pm 1.3$ & $1.0 \pm 1.1$ & 0.009 \\
\hline Median (range) & $0.9(0.2-4.1)$ & $2.3(0.5-3.2)$ & $0.8(-2.3-2.7)$ & & $2.0(0.9-5.4)$ & $0.9(-1.1-3.4)$ & \\
\hline \multicolumn{8}{|c|}{ Non-dominant hand } \\
\hline Mean \pm SD & $1.3 \pm 0.9$ & $2.2 \pm 0.7$ & $0.7 \pm 1.2$ & 0.071 & $2.1 \pm 1.0$ & $0.8 \pm 1.2$ & 0.050 \\
\hline Median (range) & $0.9(0.2-3.2)$ & $2.3(1.4-3.2)$ & $0.5(-0.9-2.5)$ & & $2.0(0.7-3.6)$ & $0.5(-1.1-3.4)$ & \\
\hline \multicolumn{8}{|l|}{ Kapandji score/10 } \\
\hline \multicolumn{8}{|l|}{ Dominant hand } \\
\hline Mean \pm SD & $8.0 \pm 1.4$ & $8.3 \pm 1.6$ & $0.3 \pm 1.0$ & 0.380 & $8.4 \pm 1.7$ & $0.5 \pm 0.9$ & 0.111 \\
\hline Median (range) & $8.0(5.0-10.0)$ & $9.0(5.0-10.0)$ & $0.0(-1.0-2.5)$ & & $9.3(5.0-10.0)$ & $0.0(-1.0-2.5)$ & \\
\hline \multicolumn{8}{|c|}{ Non-dominant hand } \\
\hline Mean \pm SD & $8.5 \pm 1.2$ & $8.4 \pm 1.6$ & $-0.1 \pm 0.7$ & 1.000 & $8.8 \pm 1.3$ & $0.4 \pm 0.7$ & 0.188 \\
\hline Median (range) & $9.0(6.0-10.0)$ & $9.0(5.0-10.0)$ & $0.0(-1.0-1.5)$ & & $9.0(6.0-10.0)$ & $0.0(-0.5-2.0)$ & \\
\hline \multicolumn{8}{|c|}{ 1st corner distance (mm) } \\
\hline \multicolumn{8}{|c|}{ Dominant hand } \\
\hline Mean \pm SD & $105.6 \pm 24.7$ & $112.9 \pm 29.2$ & $7.3 \pm 8.2$ & 0.010 & $118.9 \pm 31.2$ & $13.3 \pm 11.6$ & 0.002 \\
\hline Median (range) & $112.0(57-142)$ & $118.5(57-154)$ & $5.5(-6-23)$ & & $124.0(58-178)$ & $10.0(-3-36)$ & \\
\hline \multicolumn{8}{|c|}{ Non-dominant hand } \\
\hline Mean \pm SD & $115.8 \pm 24.5$ & $122.3 \pm 20.9$ & $6.5 \pm 9.8$ & 0.042 & $132.8 \pm 28.0$ & $17.0 \pm 14.7$ & 0.002 \\
\hline Median (range) & $118.5(65-152)$ & $121.5(88-155)$ & $4.5(-8-23)$ & & $137.0(95-190)$ & $15.5(-4-38)$ & \\
\hline \multicolumn{8}{|c|}{ Sum of corners distances $(\mathrm{mm})$} \\
\hline \multicolumn{8}{|l|}{ Dominant hand } \\
\hline Mean \pm SD & $133.9 \pm 18.5$ & $131.2 \pm 20.7$ & $1.0 \pm 13.7$ & 0.822 & $140.2 \pm 26.6$ & $9.8 \pm 15.3$ & 0.073 \\
\hline Median (range) & $130.5(110-168)$ & $131.0(94-169)$ & $2.0(-18-28)$ & & $131.0(105-188)$ & $5.5(-7-36)$ & \\
\hline \multicolumn{8}{|c|}{ Non-dominant hand } \\
\hline Mean \pm SD & $132.1 \pm 24.6$ & $133.7 \pm 29.4$ & $4.5 \pm 6.8$ & 0.052 & $137.9 \pm 27.0$ & $8.3 \pm 5.1$ & $<0.001$ \\
\hline Median (range) & $139.0(73-158)$ & $139.5(64-166)$ & $6.0(-9-13)$ & & $147.0(77-177)$ & $10.0(1-19)$ & \\
\hline \multicolumn{8}{|c|}{ Sum of Pad/DPL distance $(\mathrm{mm})$} \\
\hline \multicolumn{8}{|c|}{ Dominant hand } \\
\hline Mean \pm SD & $52.0 \pm 46.5$ & $47.3 \pm 43.8$ & $-4.8 \pm 18.6$ & 0.395 & $43.9 \pm 42.7$ & $-8.1 \pm 16.2$ & 0.111 \\
\hline Median (range) & $49.5(0-160)$ & $45.0(0-115)$ & $-2.5(-45-23)$ & & $36.5(0-125)$ & $-4.0(-35-19)$ & \\
\hline \multicolumn{8}{|c|}{ Non-dominant hand } \\
\hline Mean \pm SD & $48.1 \pm 54.5$ & $46.8 \pm 52.0$ & $-1.3 \pm 19.6$ & 0.830 & $37.8 \pm 43.0$ & $-10.3 \pm 20.6$ & 0.109 \\
\hline Median (range) & $32.0(0-144)$ & $38.5(0-160)$ & $-0.5(-43-28)$ & & $15.0(0-111)$ & $-2.0(-41-22)$ & \\
\hline \multicolumn{8}{|c|}{$\begin{array}{l}\text { Bold values correspond to the } p \text { value columns. } p \leq 0.05 \text { (2-sided) was considered significant. } \\
\text { Data are mean } \pm S D \text {, median (minimum-maximum). } \\
\text { * } p \text { Value from baseline to M2. } \\
\text { tp Value from baseline to M6. } \\
\text { CHFS, Cochin Hand Functional Scale; DPL, Distal Palmar Line. }\end{array}$} \\
\hline
\end{tabular}

3 months before entry and all during the trial. Thus, SVF injection was the sole therapeutic change within the study period, allowing evaluation of safety, tolerability and therapeutic effects.

SVF treatment was associated with an $\sim 50 \%$ improvement of CHFS, RCS, hand pain and global disability at 2 and 6 months. Although these scales are clearly subjective evaluations, they remain valid instruments when looking at change within individuals. These endpoints were complemented by improvement of objective criteria such as grip and finger's strength, corners distances and fingers' circumference, and nailfold capillaroscopy data. When analyses were performed taking into account the cutaneous form of the disease, both patients with diffuse and limited forms benefited from the SVF injections. Kapandji and pad to distal palmar line distances did not improve during the trial but were only faintly altered at start, with little room for improvement. Altogether, these changes suggest that autologous SVF injection can counterbalance the various physiopathogenic factors involved in SSc disease. In the literature, no such large 
Table 3 Effect of autologous adipose-derived stromal vascular fraction injection on scleroderma and skin thickness

\begin{tabular}{|c|c|c|c|c|c|c|c|}
\hline & Baseline & 2 months & Change (2 months-baseline) & p Value* & 6 months & Change (6 months--baseline) & $\mathrm{p}$ Valuet \\
\hline \multicolumn{8}{|c|}{ Mean circumference of F1-F5 (ring size) } \\
\hline \multicolumn{8}{|c|}{ Dominant hand } \\
\hline Mean \pm SD & $61.9 \pm 2.2$ & $60.7 \pm 2.3$ & $-1.2 \pm 1.4$ & 0.013 & $59.8 \pm 2.4$ & $-2.1 \pm 1.1$ & $<0.001$ \\
\hline Median (range) & $61.8(58.8-66)$ & $60.8(57-65)$ & $-0.9(-4.6-0.6)$ & & $60(56-64.4)$ & $-2.3(-4.6--0.4)$ & \\
\hline \multicolumn{8}{|l|}{ Non-dominant hand } \\
\hline Mean \pm SD & $60.7 \pm 2.3$ & $59.3 \pm 1.7$ & $-1.3 \pm 1.4$ & 0.008 & $58.1 \pm 2.2$ & $-2.5 \pm 1.5$ & $<0.001$ \\
\hline Median (range) & $61(57-64.2)$ & $59(56.8-63.4)$ & $-1.2(-3.4-0.8)$ & & $58(54-62.8)$ & $-2.5(-4.8-0.4)$ & \\
\hline \multicolumn{8}{|c|}{ MRSS applied to hand/18 } \\
\hline \multicolumn{8}{|c|}{ Whole population } \\
\hline Mean \pm SD & $10.9 \pm 4.9$ & $10.0 \pm 5.3$ & $-0.9 \pm 1.6$ & 0.067 & $9.9 \pm 6.0$ & $-1.0 \pm 2.8$ & 0.246 \\
\hline Median (range) & $11.5(3-18)$ & $10.5(2-18)$ & $-0.5(-4-1)$ & & $12(1-18)$ & $-2(-5-4)$ & \\
\hline \multicolumn{8}{|l|}{ Global MRSS/51 } \\
\hline \multicolumn{8}{|l|}{ Whole population } \\
\hline Mean \pm SD & $13.9 \pm 9.8$ & $11.7 \pm 9.8$ & $-2.3 \pm 2.5$ & 0.010 & $11.5 \pm 10.1$ & $-2.4 \pm 2.8$ & 0.013 \\
\hline Median (range) & $12(3-32)$ & $9(2-29)$ & $-2(-6-3)$ & & $8.5(1-29)$ & $-2(-7-2)$ & \\
\hline \multicolumn{8}{|c|}{$\begin{array}{l}\text { Data are mean } \pm S D \text {. } \\
\text { * } p \text { Value from baseline to } M 2 . \\
t p \text { Value from baseline to } M 6 .\end{array}$} \\
\hline
\end{tabular}

Table 4 Effect of autologous adipose-derived stromal vascular fraction injection on vascular manifestations

\begin{tabular}{|c|c|c|c|c|c|c|c|}
\hline & Baseline & 2 months & Change (2 months-baseline) & p Value* & 6 months & Change (6 months-baseline) & $\mathrm{p}$ Valuet \\
\hline \multicolumn{8}{|l|}{$\mathrm{RCS} / 10$} \\
\hline Mean \pm SD & $7.2 \pm 0.9$ & $4.5 \pm 1.9$ & $-2.7 \pm 2.2$ & $<0.001$ & $2.9 \pm 1.4$ & $-4.3 \pm 2.1$ & $<0.001$ \\
\hline Median (range) & $7.5(6.5-8.0)$ & $5.0(4.0-5.5)$ & $-2.0(-6.5--1.0)$ & & $3.0(2.5-3.0)$ & $-4.5(-7.0--1.5)$ & \\
\hline \multicolumn{8}{|c|}{ Hand visual analogue scale/100 } \\
\hline Mean \pm SD & $59.4 \pm 17.2$ & $21.6 \pm 17.5$ & $-37.8 \pm 28.6$ & 0.001 & $17.8 \pm 15.3$ & $-41.7 \pm 22.7$ & $<0.001$ \\
\hline Median (range) & $58.5(50.0-72.5)$ & $19.0(7.0-40.0)$ & $-39.0(-80.0-21.0)$ & & $13.6(9.0-26.0)$ & $-44.0(-80.0-10.0)$ & \\
\hline \multicolumn{8}{|l|}{ Capillaroscopy data } \\
\hline \multicolumn{8}{|c|}{ Number of capillary loops } \\
\hline \multicolumn{8}{|c|}{ Dominant hand } \\
\hline Mean \pm SD & $136.3 \pm 78.9$ & $124.1 \pm 60.1$ & $-12.2 \pm 40.9$ & 0.325 & $138.5 \pm 60.8$ & $2.3 \pm 47.9$ & 0.874 \\
\hline Median (range) & $124.0(42-298)$ & $118.0(30-226)$ & $-12.5(-98-61)$ & & $132.5(48-232)$ & $8.0(-97-92)$ & \\
\hline \multicolumn{8}{|l|}{ Non-dominant hand } \\
\hline Mean \pm SD & $159.5 \pm 96.9$ & $142.1 \pm 71.0$ & $-23.5 \pm 54.1$ & 0.181 & $152.3 \pm 75.2$ & $-11.7 \pm 42.0$ & 0.377 \\
\hline Median (range) & $122.0(45-298)$ & $139.0(42-277)$ & $-13.0(-137-33)$ & & $131.5(70-308)$ & $-10.0(-103-34)$ & \\
\hline \multicolumn{8}{|c|}{ Number of giant capillaries } \\
\hline \multicolumn{8}{|c|}{ Dominant hand } \\
\hline Mean \pm SD & $20.3 \pm 20.3$ & $17.8 \pm 18.0$ & $-2.4 \pm 8.4$ & 0.341 & $15.3 \pm 17.9$ & $-4.9 \pm 7.8$ & 0.035 \\
\hline Median (range) & $12.0(0-64)$ & $13.0(1-67)$ & $1.0(-22-7)$ & & $9.0(0-63)$ & $-1.0(-17-6)$ & \\
\hline \multicolumn{8}{|l|}{ Non-dominant hand } \\
\hline Mean \pm SD & $18.3 \pm 11.7$ & $18.2 \pm 20.5$ & $-5.5 \pm 7.9$ & & $18.1 \pm 18.6$ & $-5.1 \pm 8.9$ & 0.074 \\
\hline Median (range) & $13.0(0-39)$ & $12.5(0-77)$ & $-3.0(-20-3)$ & 0.046 & $13.0(0-72)$ & $-3.0(-23-4)$ & \\
\hline \multicolumn{8}{|c|}{ Number of dystrophic capillaries } \\
\hline \multicolumn{8}{|c|}{ Dominant hand } \\
\hline Mean \pm SD & $23.3 \pm 26.8$ & $13.0 \pm 11.6$ & $-10.3 \pm 17.9$ & 0.115 & $11.0 \pm 10.3$ & $-12.3 \pm 20.2$ & 0.047 \\
\hline Median (range) & $14.0(0-79)$ & $14.0(0-30)$ & $-2.0(-53-6)$ & & $8.5(0-29)$ & $-1.0(-57-5)$ & \\
\hline \multicolumn{8}{|l|}{ Non-dominant hand } \\
\hline Mean \pm SD & $23.2 \pm 22.8$ & $11.2 \pm 9.5$ & $-11.4 \pm 14.9$ & & $9.7 \pm 6.7$ & $-13.5 \pm 19.8$ & 0.047 \\
\hline Median (range) & $17.0(0-58)$ & $8.0(0-29)$ & $-4.0(-37-6)$ & 0.030 & $10.5(0-22)$ & $-6.0(-45-15)$ & \\
\hline \multicolumn{8}{|c|}{ Vascular suppression score } \\
\hline \multicolumn{8}{|c|}{ Dominant hand } \\
\hline Mean \pm SD & $1.7 \pm 0.8$ & $1.6 \pm 0.6$ & $-0.1 \pm 0.4$ & 0.656 & $1.5 \pm 0.7$ & $-0.2 \pm 0.3$ & 0.010 \\
\hline Median (range) & $1.9(1.0-2.3)$ & $1.5(1.1-2.1)$ & $0.0(-1.0-0.4)$ & & $1.4(0.9-2.0)$ & $-0.2(-0.4-0.0)$ & \\
\hline \multicolumn{8}{|l|}{ Non-dominant hand } \\
\hline Mean \pm SD & $1.6 \pm 0.7$ & $1.5 \pm 0.7$ & $-0.2 \pm 0.2$ & & $1.3 \pm 0.7$ & $-0.3 \pm 0.3$ & 0.003 \\
\hline Median (range) & $1.8(0.9-2.2)$ & $1.6(0.8-2.0)$ & $-0.1(-0.6-0.0)$ & 0.031 & $1.1(0.8-2.0)$ & $-0.4(-0.8-0.2)$ & \\
\hline
\end{tabular}

Bold values correspond to the $p$ value columns. $p \leq 0.05$ (2-sided) was considered significant.

Data are mean $\pm S D$, median (minimum-maximum).

*p Value from baseline to $\mathrm{M} 2$.

tp Value from baseline to M6.

Dystrophic capillaries are enlarged loops over four times the normal afferent, transition and efferent limbs width. Giant capillary loops are 10 or more times the normal width of capillary limbs. The avascular score was assessed according to Lee's method ${ }^{18}$, in which each finger was rated from 0 to 3 : grade $0=$ no avascular area; $1=0$ one or two discrete avascular areas; 2=more than two discrete avascular areas; $3=e x t e n s i v e$ and confluent avascular areas. Vascular suppression score are the mean of fingers. RCS, Raynaud's Condition Score. 
effect has been reported in patients with SSc: in the two randomised, prospective, placebo-controlled, double-blind studies (RAPIDS- $1,{ }^{6}$ RAPIDS- $2^{7}$ ), bosentan showed its efficacy on prevention of DUs, but without any significant improvement of the HAQ score.

SVF injection predominantly improved vascular peripheral manifestations such as Raynaud's phenomenon severity, DUs outcome and hand pain, the latter being in major part related to chronic vasospasm and DUs. The decrease of fingers' circumference was probably related to an improvement of finger skin oedema, but we did not measure the hand volume to differentiate oedema from fibrosis. Altogether, these effects could explain the functional improvement observed in this study. These observations also suggest that SVF may improve vasomotor tone and microvascular perfusion. This hypothesis is further substantiated by the significant reduction of avascular areas and dystrophic capillaries evaluated using nailfold capillaroscopy. SVF mainly promotes vascular repair and angiogenesis as documented in various experimental models of tissue ischaemia. ${ }^{34}$ Moreover, defective endogenous capacity for vascular repair has been involved in SSc pathogenesis. ${ }^{36}$ Thus, local supply of high amount of endothelial precursors, mesenchymal-like stem cells, pericytes and various angiogenic factors through SVF injection in damaged fingers can directly contribute to improve vascular deficiency. We found that the main characteristics of the Celution processed SVF from patients with SSc were comparable to previous reports ${ }^{37}$ and to our personal observations from healthy subjects (data not shown). Consistently, BM-MSC from patients with SSc were reported to exhibit similar phenotypic, proliferative, differentiation potential and immunosuppressive properties as their healthy counterparts. ${ }^{38}{ }^{39}$ Given the heterogeneity of SVF and the as-yet unclear mechanisms, it would be difficult to determine whether the properties critical for efficacy are impaired in SVF from patients with SSc compared with that of healthy donors.

No significant correlation was observed between the characteristics of the injected SVF and clinical outcomes. Further study in a larger cohort may help delineate the contribution of each cell subpopulation to the clinical effect.

Although this was a single-centre, phase I study in a limited number of patients and with relatively brief follow-up, it showed that this innovative treatment was not only safe and well tolerated in patients with SSc but also provided encouraging preliminary evidence of efficacy in numerous secondary endpoints. Longer-term, larger and controlled studies will be important to confirm whether this new form of cell therapy can improve the long-term prognosis of this debilitating disease. In addition, future studies may help understand the mechanisms supporting the clinical effects of SVF.

\footnotetext{
Author affiliations

${ }^{1}$ Internal Medicine Department, Assistance Publique Hôpitaux de Marseilles (APHM), Aix-Marseilles University, Marseilles, France

${ }^{2}$ Vascular Research Center Marseille, INSERM UMRS-1076, Aix-Marseilles University, Marseilles, France

${ }^{3}$ CIC- CPCET, Assistance Publique Hôpitaux de Marseilles (AP-HM), Aix-Marseilles University, Marseilles, France

${ }^{4}$ Plastic Surgery Department, Assistance Publique Hôpitaux de Marseilles (AP-HM), Aix-Marseilles University, Marseilles, France

${ }^{5}$ Cell Therapy Facility \& Inserm CBT 510, Institut Paoli Calmettes, Marseilles, France ${ }^{6}$ Department of Radiology, Assistance Publique Hôpitaux de Marseille (AP-HM), Aix Marseilles University, Marseilles, France

${ }^{7}$ Délégation de la Recherche et de I'Innovation, Assistance Publique Hôpitaux de Marseilles (AP-HM), Marseilles, France

${ }^{8}$ Department of Dermatology, Assistance Publique Hôpitaux de Marseilles (AP-HM), Aix-Marseilles University, Marseilles, France
}

${ }^{9}$ Culture and Cell Therapy Laboratory, INSERM CBT 510, Assistance Publique Hôpitaux de Marseilles (AP-HM), Aix-Marseilles University, Marseilles, France

${ }^{10}$ Hematology and Vascular Biology Laboratory, Assistance Publique Hôpitaux de Marseilles (AP-HM), Aix-Marseilles University, Marseilles, France

Acknowledgements We would like to thank the French Scleroderma Research Group for financial support. We also thank the Fondation de l'Avenir, Estelle Charles-Baumel, who contributed to data management, Didier Samson, who anaesthetised the patients, and Joelle Micallef, for her advices in the redaction of the manuscript.

Contributors All authors contributed to review of this manuscript. BG, $A D, E J, F S$, JV and GM designed the protocol. SM did the dermatological assessment, NC did the Doppler US and X-ray and both helped analysing the data. AB did the physiotherapist assessments. $B G$ and $A D$ did the recruitment of patients and the inpatient and outpatient care. GM, PSN and DC performed the surgical procedure. FS, JV, LG and LA were in charge of the SVF preparation and packaging of the SVF for injection. They also did the characterisation and the quality controls of the SVF. JCR monitored the trial. EJ and RT performed the statistical analyses, reviewed charts and data and helped in interpretation of the findings. JRH was at the head of the independent survey committee of the study. CC criticised and reviewed the manuscript.

\section{Competing interests None.}

Ethics approval Local Ethic Committee and French Drug Agency.

Provenance and peer review Not commissioned; externally peer reviewed.

Open Access This is an Open Access article distributed in accordance with the Creative Commons Attribution Non Commercial (CC BY-NC 4.0) license, which permits others to distribute, remix, adapt, build upon this work non-commercially, and license their derivative works on different terms, provided the original work is properly cited and the use is non-commercial. See: http://creativecommons.org/ licenses/by-nc/4.0/

\section{REFERENCES}

1 Guillevin L, Hunsche E, Denton $\mathrm{CP}$, et al.; DUO Registry Group. Functional impairment of systemic scleroderma patients with digital ulcerations: results from the DUO Registry. Clin Exp Rheumatol 2013;31:71-80.

2 Hachulla E, Clerson P, Launay D, et al. Natural history of ischemic digital ulcers in systemic sclerosis: single-center retrospective longitudinal study. J Rheumatol 2007:34:2423-30.

3 Sandqvist G, Eklund M. Hand Mobility in Scleroderma (HAMIS) test: the reliability of a novel hand function test. Arthritis Care Res 2000;13:369-74.

4 Avouac J, Guerini H, Wipff J, et al. Radiological hand involvement in systemic sclerosis. Ann Rheum Dis 2006:65:1088-92.

5 Kowal-Bielecka 0, Landewé R, Avouac J, et al. EULAR recommendations for the treatment of systemic sclerosis: a report from the EULAR Scleroderma Trials and Research group (EUSTAR). Ann Rheum Dis 2009;68:620-8.

6 Korn JH, Mayes M, Matucci Cerinic M, et al. Digital ulcers in systemic sclerosis: prevention by treatment with bosentan, an oral endothelin receptor antagonist. Arthritis Rheum 2004;50:3985-93.

7 Matucci-Cerinic M, Denton CP, Furst DE, et al. Bosentan treatment of digital ulcers related to systemic sclerosis: results from the RAPIDS-2 randomised, double-blind, placebo-controlled trial. Ann Rheum Dis 2011;70:32-8.

8 Brueckner CS, Becker MO, Kroencke T, et al. Effect of sildenafil on digital ulcers in systemic sclerosis: analysis from a single centre pilot study. Ann Rheum Dis 2010:69:1475-8

9 Wigley FM, Seibold JR, Wise RA, et al. Intravenous iloprost treatment of Raynaud's phenomenon and ischemic ulcers secondary to systemic sclerosis. J Rheumatol 1992;19:1407-14.

10 Pope JE, Bellamy N, Seibold JR, et al. A randomized, controlled trial of methotrexate versus placebo in early diffuse scleroderma. Arthritis Rheum 2001:44:1351-8

11 Jewett $L R$, Hudson M, Malcarne VL, et al. Sociodemographic and disease correlates of body image distress among patients with systemic sclerosis. PLoS One 2012;7: e33281.

12 Traverse JH, Henry TD, Ellis SG, et al. Effect of intracoronary delivery of autologous bone marrow mononuclear cells 2 to 3 weeks following acute myocardial infarction on left ventricular function: the LateTIME randomized trial. JAMA 2011:306:2110-19.

13 Matoba S, Tatsumi T, Murohara T, et al. Long-term clinical outcome after intramuscular implantation of bone marrow mononuclear cells (Therapeutic Angiogenesis by Cell Transplantation [TACT] trial) in patients with chronic limb ischemia. Am Heart J 2008;156:1010-18.

14 Hare JM, Fishman JE, Gerstenblith $\mathrm{G}$, et al. Comparison of allogeneic vs autologous bone marrow-derived mesenchymal stem cells delivered by transendocardial injection in patients with ischemic cardiomyopathy: the POSEIDON randomized trial. JAMA 2012;308:2369-79. 
15 Lu D, Chen B, Liang Z, et al. Comparison of bone marrow mesenchymal stem cells with bone marrow-derived mononuclear cells for treatment of diabetic critical limb ischemia and foot ulcer: a double-blind, randomized, controlled trial. Diabetes Res Clin Pract 2011;92:26-36.

16 Zuk PA, Zhu M, Ashjian P, et al. Human adipose tissue is a source of multipotent stem cells. Mol Biol Cell 2002;13:4279-95.

17 Kapur SK, Katz AJ. Review of the adipose derived stem cell secretome. Biochimie. 2013:95:2222-8.

18 Leto Barone AA, Khalifian S, Lee WP, et al. Immunomodulatory effects of adipose-derived stem cells: fact or fiction?. Biomed Res Int 2013;2013:383685.

19 Bura A, Planat-Benard V, Bourin P, et al. Phase I trial: the use of autologous cultured adipose-derived stroma/stem cells to treat patients with non-revascularizable critical limb ischemia. Cytotherapy 2014;16:245-57.

20 Scuderi N, Ceccarelli S, Onesti MG, et al. Human adipose-derived stromal cells for cell-based therapies in the treatment of systemic sclerosis. Cell Transplant 2013;22:779-95

21 Subcommittee for Scleroderma Criteria of the American Rheumatism Association Diagnostic and Therapeutic Criteria Committee. Preliminary criteria for the classification of systemic sclerosis (scleroderma). Arthritis Rheum 1980;23:581-90.

22 LeRoy EC, Black C, Fleischmajer R, et al. Scleroderma (systemic sclerosis): classification,subsets and pathogenesis. J Rheumatol 1988;15:202-5.

23 Van den Hoogen F, Khanna D, Fransen J, et al. 2013 classification criteria for systemic sclerosis: an American College of Rheumatology/European League against Rheumatism collaborative initiative. Arthritis Rheum 2013:65:2737-47.

24 Rannou F, Poiraudeau S, Berezné A, et al. Assessing disability and quality of life in systemic sclerosis: construct validities of the Cochin Hand Function Scale, Health Assessment Questionnaire (HAQ), Systemic Sclerosis HAQ, and Medical Outcomes Study 36-Item Short Form Health Survey. Arthritis Rheum 2007:57:94-102.

25 Bourin $P$, Bunnell BA, Casteilla $L$, et al. Stromal cells from the adipose tissue-derived stromal vascular fraction and culture expanded adipose tissue-derived stromal/stem cells: a joint statement of the International Federation for Adipose Therapeutics and Science (IFATS) and the International Society for Cellular Therapy (ISCT). Cytotherapy 2013;15:641-8.

26 Kapandji A. Clinical test of apposition and counter-apposition of the thumb Cotation clinique de l'opposition et de la contre-opposition du pouce. Ann Chir Main 1986;5:67-73.
27 Mathiowetz V, Kashman N, Volland G, et al. Grip and pinch strength: normative data for adults. Arch Phys Med Rehabil 1985;66:69-74.

28 Lee $\mathrm{P}$, Leung FY, Alderdice $C$, et al. Nailfold capillary microscopy in the connective tissue diseases: a semiquantitative assessment. J Rheumatol 1983;10:930-8.

29 Clements PJ, Lachenbruch PA, Seibold JR, et al. Skin thickness score in systemic sclerosis: an assessment of interobserver variability in 3 independent studies. J Rheumatol 1993;20:1892-6.

30 Georges C, Chassany O, Mouthon L, et al. Validation of French version of the Scleroderma Health Assessment Questionnaire (SSC HAQ). Clin Rheumatol 2005:24:3-10.

31 Katz AJ, Tholpady A, Tholpady SS, et al. Cell surface and transcriptional characterization of human adipose-derived adherent stromal (hADAS) cells. Stem Cells 2005;23:412-23.

32 Lee SH, Lee EJ, Lee SY, et al. The effect of adipose stem cell therapy on pulmonary fibrosis induced by repetitive intratracheal bleomycin in mice. Exp Lung Res 2014; 40:117-25

33 Ikegame $Y$, Yamashita $\mathrm{K}$, Hayashi $\mathrm{S}$, et al. Comparison of mesenchymal stem cells from adipose tissue and bone marrow for ischemic stroke therapy. Cytotherapy 2011;13:675-85.

34 Rehman J, Traktuev D, Li J, et al. Secretion of angiogenic and antiapoptotic factors by human adipose stromal cells. Circulation 2004;109:1292-8.

35 Nakagami H, Maeda K, Morishita R, et al. Novel autologous cell therapy in ischemic limb disease through growth factor secretion by cultured adipose tissue-derived stromal cells. Arterioscler Thromb Vasc Biol 2005;25:2542-7.

36 Matucci-Cerinic M, Kahaleh B, Wigley FM. Review: evidence that systemic sclerosis is a vascular disease. Arthritis Rheum 2013:65:1953-62.

37 Lin K, Matsubara Y, Masuda Y, et al. Characterization of adipose tissue-derived cells isolated with the Celution system. Cytotherapy 2008;10:417-26.

38 Larghero J, Farge D, Braccini A, et al. Phenotypical and functional characteristics of in vitro expanded bone marrow mesenchymal stem cells from patients with systemic sclerosis. Ann Rheum Dis 2008;67:443-9.

39 Cipriani P, Di Benedetto P, Liakouli V, et al. Mesenchymal stem cells (MSCs) from scleroderma patients (SSC) preserve their immunomodulatory properties although senescent and normally induce T regulatory cells (Tregs) with a functional phenotype: implications for cellular-based therapy. Clin Exp Immunol 2013; 173:195-206. 